\title{
Concurrent activation of two task sets by implicit and probabilistic contextual cues
}

\author{
Linda E. L. Wei ${ }^{1,{ }^{*}} \&$ Nick Yeung ${ }^{1}$ \\ ${ }^{1}$ Department of Experimental Psychology, University of Oxford, Oxford, UK \\ *I.wei@magd.oxon.org
}

\begin{abstract}
Real-world settings impose constantly changing demands on cognition and behaviour. These task demands can often be predicted by the context, and the implicit learning of these probabilistic contexttask associations may enhance task performance. While previous studies have focused on how task cues that are either probabilistic or implicit affect task-switching performance, the present study investigated how people learn and use contextual cues that are both implicit and probabilistic within a cued task-switching design. Participants showed response speed benefits when engaging in tasks that were predicted to be more likely by a preceding contextual cue. However, this probabilistic cueing effect was only seen when specific contextual cues were associated with task probabilities (Experiment 2), and not when contextual categories were associated with task probabilities (Experiment 1). The findings provide support for automatic activation of multiple task sets; a model of multiple concurrent task set activation and representation is proposed. Taken together, our findings suggest that people can implicitly learn probabilistic associations between specific contexts and tasks, and can use information from contexts to guide adaptive behaviour in dynamic environments.
\end{abstract}

\section{Introduction}

The environments we live in are dynamic and uncertain. These constantly changing demands on cognition and behaviour are often cued by stimuli present in the environment. A steady green traffic light at an upcoming intersection, for instance, signals to drivers to maintain speed and drive through. But if no pedestrians are crossing in that direction and the queue of cross-flow traffic is long, experienced drivers will often prepare or begin to slow down in anticipation of the green light turning yellow, sometimes without even being fully cognizant of the contextual cues that have led to their response. This kind of 'situational awareness' is usually associated with experience, and involves the ability to rapidly extract, integrate, assess, and act upon meaningful task-relevant information from the context (McGuinness \& Dawson, 2005; O'Brien \& O'Hare, 2007). Previous research has primarily focused on the perceptual and attentional mechanisms of situational awareness (Chun \& Jiang, 1998; Goujon, 2011; Kunar, Flusberg, \& Wolfe, 2006). Less frequently addressed are the contextually influenced task set activation and switching mechanisms that may underlie situational awareness and adaptive behaviour.

Context refers to the specific spatial and temporal configuration of environmental stimuli under which the task is carried out (Norman \& Shallice, 1980; 1986; Rogers \& Monsell, 1995). Contexts inform cognitive control over behaviour because they are frequently predictive of the response required. On their own, environmental stimuli are sources of uncertainty, as they may be irrelevant or afford multiple possible responses (Crump, Vaquero, \& Miliken, 2008; Mayr \& Bryck, 2005; 2006). In context, however, the appropriate response to these multivalent stimuli often becomes clear, as different contexts in which the stimuli occur are associated with different probabilities of specific tasks and their corresponding responses (Leue, Rodilla, \& Beauducel, 2015; Lindenberger \& Mayr, 2014). Learning these probabilistic associations between contexts and tasks makes it possible to more rapidly select and activate the relevant task set—an abstract procedural programme or schema of response-guiding rules-and subsequently configure cognitive processes to address task demands and execute the response 
(Arbuthnott \& Woodward, 2002; Collins \& Frank, 2013; Crump et al., 2008; Crump \& Logan, 2010; Rubin \& Koch, 2006). The implicit learning of relevant contextual cues and probabilistic context-task relationships aids the recruitment of cognitive control for responses to complex, dynamic environments (Collins \& Frank, 2013; Crump \& Logan, 2010; Lindenberger \& Mayr, 2014; Patterson et al., 2013).

The interaction between endogenously recruited (top-down) and exogenously cued (bottom-up) control mechanisms in governing such responses can be experimentally investigated using the cued task-switching paradigm (Sudevan \& Taylor, 1987; Meiran, 1996). Participants in these experiments are directed to perform a particular task by an explicit task cue before the onset of the target stimulus; tasks are presented in random order, and trials are categorised as repeat or switch trials in post hoc analyses (Logan, 2003; Mayr, 2006; Meiran, 1996). Switching from one task to another is said to incur a robust switch cost in response time (RT) and error rate, as switch trials are generally slower and less accurate than repeat trials (Monsell, 2003; Wylie \& Allport, 2000). Foreknowledge of the upcoming task (Ruthruff, Remington, \& Johnston, 2001) and a longer preparation interval (Meiran, 1996; Rogers \& Monsell, 1995) appear to reduce switch costs. Consequently, switch costs are frequently attributed to active, top-down processes like cognitive reconfiguration after the activation of a different task set by the task cue (Meiran, 1996; Rogers \& Monsell, 1995) or active inhibition of abandoned task sets (Dreisbach, Haider, \& Kluwe, 2002; Mayr \& Keele, 2000; Mayr, 2002). Alternatively, switch costs might be similarly wellaccounted for by passive, bottom-up effects: proactive interference from residual activation for the previous task set (Allport, Styles, \& Hsieh, 1994; Altmann \& Gray, 2008; Mayr \& Keele, 2000; Wylie \& Allport, 2000), priming of task set retrieval by a stimulus in the current trial (Allport \& Wylie, 2000; Waszak, Hommel, \& Allport, 2003), or priming of performance by task repetitions (Logan \& Bundesen, 2003; 2004; Mayr \& Kleigl, 2003; Ruthruff et al., 2001).

These top-down and bottom-up mechanisms in task switching may not be mutually exclusive. Previous research has suggested that the extent to which responses leverage either depends on the amount of environmental support in the experimental paradigm, namely, the extent to which cues in the environment provide the information necessary for an appropriate response (Craik, 1983; Arrington \& Logan, 2005; Lindenberger \& Mayr, 2014). In the cued task-switch paradigm, the explicit task cue provides a great deal of environmental support, rendering topdown control redundant and favouring a bottom-up account of task switching (Arrington \& Logan, 2005; Logan $\&$ Bundesen, 2003; 2004). However, the explicit and certain nature of the task cues found in these experimental paradigms may limit their ecological validity. Contextual task cues in real-world settings, like those influencing the driver's judgment of the likelihood of the green light turning yellow on them, tend to be probabilistic rather than certain. Moreover, the task probabilities these contextual cues represent-and the knowledge that these cue stimuli are informative or predictive at all-are more often learned implicitly, through repeated exposure and association, than instructed explicitly (Cleeremans, Destrebecqz, \& Boyer, 1998; Crump \& Logan, 2010; Patterson et al., 2013; Seger, 1994). It is unclear from the current literature on task-switching whether people can use contextual cues that are both probabilistic and implicit to enhance task performance, and to what extent the effects of these cues on task performance can be attributed to top-down versus bottom-up task-switching mechanisms.

In previous research, people have been found to use probabilistic task cues to enhance their task performance. The most systematic findings for this have been obtained from a variant of the cued task-switching paradigm, in which explicit and probabilistic cues of task identity (e.g., three red squares and one blue square, instructed from the outset to represent a $75 \%$ chance of the red-associated task and a $25 \%$ chance of the blue-associated task) appear a short interval before a second, certain task cue (e.g., colour and type of target stimulus) (Dreisbach et al., 2002). Dreisbach et al. found that RT increased linearly for both repeat trials and switch trials as the probability of the task, as indicated by the squares, decreased from $100 \%$ to $25 \%$. The magnitude of the cueing effect appeared to be proportional to the value of the probabilistic cue in predicting task identity. Dreisbach et al. suggested that this cueing effect might be supported by concurrent processes of intentional activation for expected task sets and automatic inhibition of irrelevant task sets. However, it is difficult to infer from their study whether probabilistic 
task cues that are implicitly learned, rather than explicitly instructed, would have the same effect on task performance, and engage the same balance of top-down and bottom-up mechanisms.

There is some experimental evidence to suggest that implicit task cues engage a similar mixture of top-down and bottom-up mechanisms. In an implicitly cued task-switching design, where participants made a spatial judgment (left/up or right/down) both explicitly cued by arrows indicating the relevant judgment dimension (left-right or up-down) and implicitly cued by the colour of the target stimulus that appeared onscreen before the arrows, Rubin and Koch (2006) found that the effect of implicit cue validity was dependent upon the cue-task interval (CTI): a RT cost of implicit cue invalidity was only observed when CTI was short $(100 \mathrm{~ms})$. The researchers suggested that implicit and explicit task cues concurrently activate associated task sets via bottom-up and top-down processes, respectively. When the task set activated by bottom-up mechanisms does not match the task set activated by top-down mechanisms (e.g., when the implicit cue is followed by an unpredicted task), an interference effect is observed. However, the interference effect appears to be limited to trials with short CTI. Rubin and Koch postulated that, once the task set activated by top-down processes reaches an activation threshold, the corresponding response is executed regardless of competing bottom-up influences. Interference is only seen when CTI is short because top-down task set activation has yet to reach threshold in those trials; once the threshold is reached after a longer CTI, task sets activated by bottom-up processes no longer influence task performance. However, Rubin and Koch's experimental design may have attenuated bottom-up effects relative to contextual cues encountered in real-world settings. In these experiments, the task probabilities reversed every three blocks. If implicit cue-task association learning drives the bottom-up activation of task sets associated with the implicit cue (e.g., Forrest, Monsell, \& McLaren, 2014; Meier, Lea, \& McLaren, 2016), then a consistent cue-task probability would be fundamentally important for establishing a strong bottom-up activation effect. Participants in Rubin and Koch's experiments may not have had enough time to learn the associations to use the implicit cue effectively; upon task probability reversal, they might have even dismissed the informational value of the implicit cue altogether.

Looking back on the task-switching literature to date, it appears that research has been conducted on how people learn and use implicit task cues, and probabilistic task cues, but not on how people might use cues, like those embedded in the context of the task, that are both implicit and probabilistic. In the present study, we sought to investigate the influence of implicit and probabilistic contextual information on task performance, using a modified version of Rubin and Koch's implicitly cued task-switching paradigm. In our design, scenic images that appeared before and remained in the background to a monochromatic target stimulus acted as context with informational value. Like the probabilistic task cues in Dreisbach et al. (2002), these background images were consistently associated with specific relative probabilities of the two possible tasks in each experiment, but unlike in Dreisbach et al. (2002), participants were not instructed that the images were task-relevant, or that cue-task associations were present and consistent. Therefore, any learning of associations between the images and task probabilities would be implicit.

In conducting our study, we had three general aims. Our first aim was to test whether participants could implicitly learn stable cue-task associations. We hypothesized that cue-task association learning would improve participants' speed and accuracy of response to tasks indicated as more likely by the preceding cue. Existing literature on unconsciously primed task-switching (e.g., Manly et al., 2014) also suggested that this cueing effect would be dissociable from explicit awareness of cues and cue-task associations. As a secondary aim, we also wanted to compare the effects of more abstract (i.e., between image category and task probability) and more concrete (i.e., between a specific image and task probability) cue-task associations. We suspected that abstract cue-task associations would be more difficult to learn, and that 'online' use of these cues in contextual control would be hindered by greater amount of processing required to interpret the task probability information conveyed by these cues. Thus, we expected abstract cue-task associations to produce weaker cueing effects than concrete cue-task associations. Finally, our study aimed to assess the relative contributions of top-down and bottom-up mechanisms in any cueing effects. Since our design held cue-task probabilities constant throughout the experiment, we hypothesized that bottom-up mechanisms would be more strongly activated in our design than in Rubin and 
Koch's original design. Consequently, we expected stronger activation for competing task sets possibly manifesting in (i) greater switch costs for uncertain cues (i.e., associated with both task sets to some extent) than certain cues (i.e., associated with only one task set), and (ii) response speed and switch cost reductions at long CTIs relative to short CTIs for low-probability events.

\section{Experiment 1}

This experiment used a cued task-switching paradigm to test whether people could implicitly learn abstract probabilistic cue-task associations, and whether this led to facilitation or interference effects in task-switching performance. In the experiment, participants switched between two spatial discrimination tasks. The task to be completed on a trial was first implicitly cued by the background image (contextual cue) that preceded the target stimulus: the type of image (contextual category) was associated with distinct probabilities for each of the two tasks to appear. A short duration (CTI) after the onset of the contextual cue, the task was indicated explicitly by the colour of a frame around the image, which appeared at the same time as the target stimulus.

\section{Method}

Participants. Eighteen fluently English-speaking volunteers recruited from the undergraduate and postgraduate student population at the University of Oxford took part in the experiment ( 14 female; $M=20.61$ years, $S D=$ .85 years, range 19-22 years). All participants had normal or corrected-to-normal vision, gave written informed consent prior to testing, and were reimbursed for their time with $£ 10$.

Apparatus and stimuli. PsychoPy v.1.82.01 (Pierce, 2007) was used to present the experiment and create all stimuli in the experiment except the contextual cue. The background image acting as the contextual cue (740 $\mathrm{x}$ 740 pixels, $30 \%$ opacity, centrally presented) appeared onscreen at the beginning of each trial. The specific image shown belonged to one of five categories ${ }^{1}$, with each category predicting different probabilities of the two tasks ${ }^{2}$. Sixty different images were shown exactly once during each experimental block (one image per trial, twelve images per image category).

After a variable CTI, the target stimulus and coloured frame appeared simultaneously, overlaid on the background image (Figure 1a). The target stimulus was a white square $(100 \times 100$ pixels) presented at the centre of one of four quadrants demarcated by the grid. The coloured frame (10 pixels thick) around the background image appeared at the same time as the target stimulus and grid. A red frame signalled an up-down task, where participants indicated whether the target was the in upper or lower part of the grid. A blue frame signalled a leftright task, where participants indicated whether the target was the in the left or right part of the grid.

Procedure. The experiment was conducted in a single session lasting 60 minutes. At the start of each trial (Figure $1 b$ ), the contextual cue appeared onscreen and remained onscreen throughout the trial; task stimuli (i.e., target stimulus, grid, and coloured frame around the image) then appeared after a short delay. The interval between the onset of the contextual cue and the onset of the task stimuli (CTI) was $100 \mathrm{~ms}, 550 \mathrm{~ms}$, or $1100 \mathrm{~ms}$ in length. The presence or significance of the contextual cue was not mentioned to the participant by the experimenter at any point during the experiment. Responses were made with the index finger of both hands by pressing keys on a QWERTY keyboard ('m' for 'right' and 'down' responses; ' $\mathrm{t}$ ' for 'left' and 'up' responses). RTs were measured from the onset of task stimuli. Following the participant's response, a blank grey screen was shown for an interval (response-cue interval; RCI) that lasted $1400 \mathrm{~ms}, 950 \mathrm{~ms}$, or $400 \mathrm{~ms}$, respectively. This ensured that the total time

\footnotetext{
${ }^{1}$ During the practice block, four images not belonging to one of these five categories were cycled fifteen times each. These four images did not appear during later experimental blocks.

${ }^{2}$ The image categories and their corresponding task probabilities were: $a$ ) mountains [ $100 \%$ for left-right], $b$ ) forests [75\% for left-right, $25 \%$ for up-down], $c$ ) fields [50\% for left-right, $50 \%$ for up-down], $d$ ) villages [25\% for left-right, $75 \%$ for updown], and $e$ ) cities [100\% for up-down].
} 
a
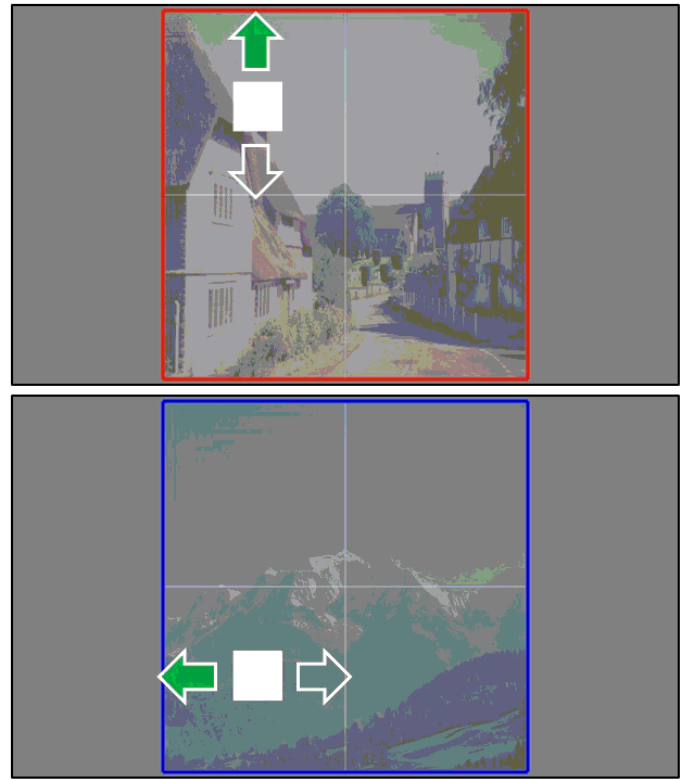

b

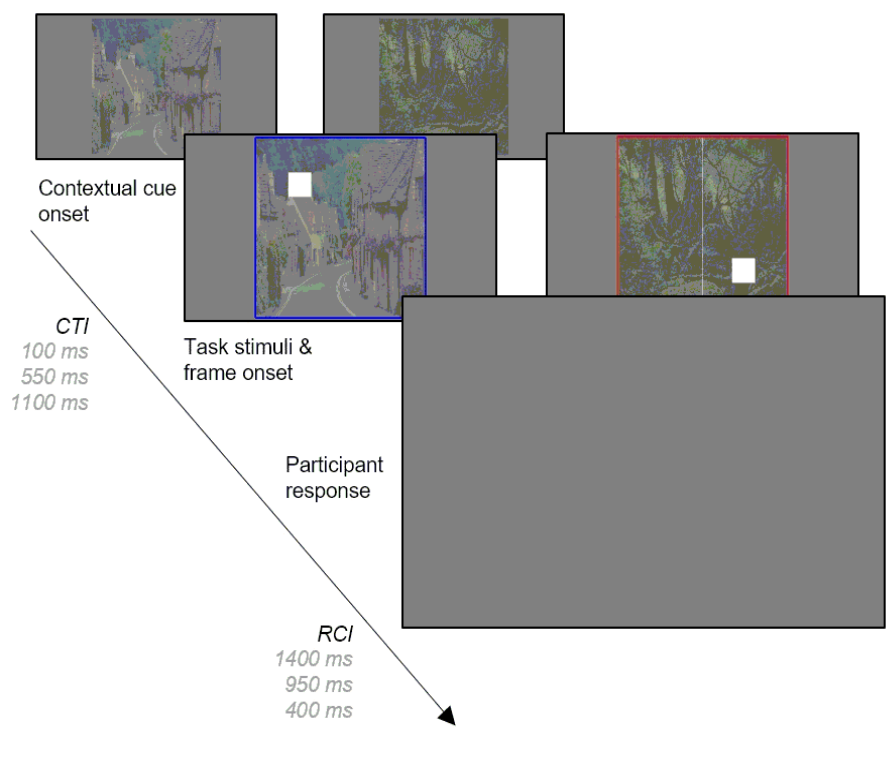

Figure 1. Spatial discrimination task in Experiment 1.

a. The two frame colours and their corresponding spatial discrimination dimensions. The green arrow (not shown in experiment) indicates what the correct response to the spatial discrimination task cued by the frame colour would be. b. Format of trials in Experiment 1. The CTI and RCI are variable intervals (all three levels used in the experiment are shown in grey).

between the participant's response on one trial and the onset of the spatial discrimination task on the following trial remained constant at $1500 \mathrm{~ms}$.

After a practice block of 60 trials, participants completed 15 experimental blocks (60 trials each). No immediate post-trial feedback was given, but participants were presented with a summary of their mean RT and accuracy at the end of each block. At the end of all experimental blocks, participants were asked by the experimenter to identify five categories of images they had seen during the experiment; participants were also asked whether they noticed an association between the images and the tasks. Following these questions, participants performed a surprise cue recognition memory task, in which they were shown an image onscreen and asked to indicate if they had seen the image during the experiment. Participants registered their response by clicking on a six-point familiarity scale located below the image $(-3=$ absolutely certain not seen; $-2=$ moderately certain not seen; -1 = somewhat certain not seen; 1 = somewhat certain seen; 2 = moderately certain seen; $3=$ absolutely certain seen). There were 90 images shown (60 seen during the experiment, 30 unseen). Fifteen of the unseen images belonged to the same categories as the background images observed during the experiment, and the other fifteen belonged to different categories. Recognition memory score (possible range: 0 to 1) was calculated using the formula described in Supplementary Material (S1).

\section{Results}

The first trial of each block (1.7\% of total) was excluded from both response time (RT) and error rate analyses. In addition, for RT analyses, trials on which errors were made ( $4.9 \%$ of total) and RT outliers ( $>3 S D$ from the mean or under $300 \mathrm{~ms})(1.7 \%$ of total) were identified for each participant and excluded. Mean RTs, error rates, and their respective switch costs are summarized in Table 1 and 2, respectively.

RTs and error rates were analyzed in separate 4 (probability: $0.25,0.50,0.75,1.00) \times 2$ (trial type: repeat, switch) $\times 3$ (CTI: $100,550,1100)$ repeated measures ANOVA with alpha levels of .05 using SPSS v.22. Differences in RT and error rates across all levels of all independent variables are illustrated in Figure 2a-d. 
Table 1

Mean RT (ms) as a function of trial type, CTI, and probability in Experiment 1

\begin{tabular}{|c|c|c|c|c|c|c|}
\hline \multirow[b]{3}{*}{ CTI } & \multirow[b]{3}{*}{ Probability } & \multicolumn{4}{|c|}{ Trial type } & \multirow[b]{3}{*}{ Switch cost } \\
\hline & & \multicolumn{2}{|c|}{ Repeat } & \multicolumn{2}{|c|}{ Switch } & \\
\hline & & $M$ & $S E$ & $M$ & $S E$ & \\
\hline \multirow[t]{4}{*}{100} & 0.25 & 734 & 29 & 820 & 22 & 86 \\
\hline & 0.50 & 725 & 27 & 807 & 20 & 82 \\
\hline & 0.75 & 709 & 25 & 831 & 21 & 122 \\
\hline & 1.00 & 739 & 27 & 842 & 28 & 103 \\
\hline \multirow[t]{4}{*}{550} & 0.25 & 656 & 25 & 799 & 27 & 143 \\
\hline & 0.50 & 675 & 25 & 805 & 24 & 130 \\
\hline & 0.75 & 680 & 26 & 803 & 30 & 123 \\
\hline & 1.00 & 675 & 25 & 764 & 24 & 89 \\
\hline \multirow[t]{4}{*}{1100} & 0.25 & 712 & 33 & 778 & 34 & 66 \\
\hline & 0.50 & 681 & 26 & 768 & 20 & 87 \\
\hline & 0.75 & 694 & 35 & 767 & 30 & 73 \\
\hline & 1.00 & 701 & 26 & 776 & 29 & 75 \\
\hline
\end{tabular}

Table 2

Mean error rate (\% incorrect) as a function of trial type, CTI, and probability in Experiment 1

\begin{tabular}{|c|c|c|c|c|c|c|}
\hline \multirow[b]{3}{*}{ CTI } & \multirow[b]{3}{*}{ Probability } & \multicolumn{4}{|c|}{ Trial type } & \multirow[b]{3}{*}{ Switch cost } \\
\hline & & \multicolumn{2}{|c|}{ Repeat } & \multicolumn{2}{|c|}{ Switch } & \\
\hline & & $M$ & $S E$ & $M$ & $S E$ & \\
\hline \multirow[t]{4}{*}{100} & 0.25 & 3.9 & 1.4 & 6.2 & 2.1 & 2.3 \\
\hline & 0.50 & 2.6 & 0.6 & 9.6 & 1.7 & 7.0 \\
\hline & 0.75 & 3.2 & 0.9 & 6.7 & 1.2 & 3.5 \\
\hline & 1.00 & 3.4 & 0.7 & 5.7 & 0.9 & 2.3 \\
\hline \multirow[t]{4}{*}{550} & 0.25 & 2.9 & 1.2 & 6.3 & 1.9 & 3.4 \\
\hline & 0.50 & 3.0 & 1.0 & 7.3 & 1.6 & 4.3 \\
\hline & 0.75 & 3.4 & 0.7 & 5.7 & 1.3 & 2.3 \\
\hline & 1.00 & 4.6 & 1.0 & 7.6 & 1.4 & 3.0 \\
\hline \multirow[t]{4}{*}{1100} & 0.25 & 3.5 & 1.2 & 3.1 & 0.9 & -0.4 \\
\hline & 0.50 & 3.7 & 0.8 & 6.3 & 1.5 & 2.6 \\
\hline & 0.75 & 3.7 & 0.9 & 5.2 & 0.9 & 1.5 \\
\hline & 1.00 & 4.2 & 1.1 & 6.2 & 1.0 & 2.0 \\
\hline
\end{tabular}

As expected in a task-switching paradigm, robust switch costs were observed for both RT and error rates. Participants responded more slowly on switch trials $(797 \mathrm{~ms})$ than on repeat trials $(698 \mathrm{~ms}), F(1,17)=61.68, p<$ $.001, \eta^{2}=.78$, and they also made more errors on switch trials $(6.3 \%)$ than repeat trials $(3.5 \%), F(1,17)=22.41$, $p<.001, \eta^{2}=.57$. In addition, a significant main effect of CTI was observed: responses were similar in accuracy, $p=.367$, but were significantly slower, $F(1.46,24.86)=33.04, p<.001, \eta^{2}=.66$ (Greenhouse-Geisser corrected), for the shortest CTI of $100 \mathrm{~ms}(776 \mathrm{~ms})$ than for either of the other two CTIs. The difference between the RTs for medium (732 ms) and long CTIs $(735 \mathrm{~ms})$ was not significant. The interaction effect of trial type and CTI also approached significance for both RT, $p=.075$, and error rate, $p=.060$. RT and error rate switch costs decreased slightly but nonlinearly with increasing CTI; this was due to a decrease in RT and error rate for switch trials, as seen in Figure 2e-f. 

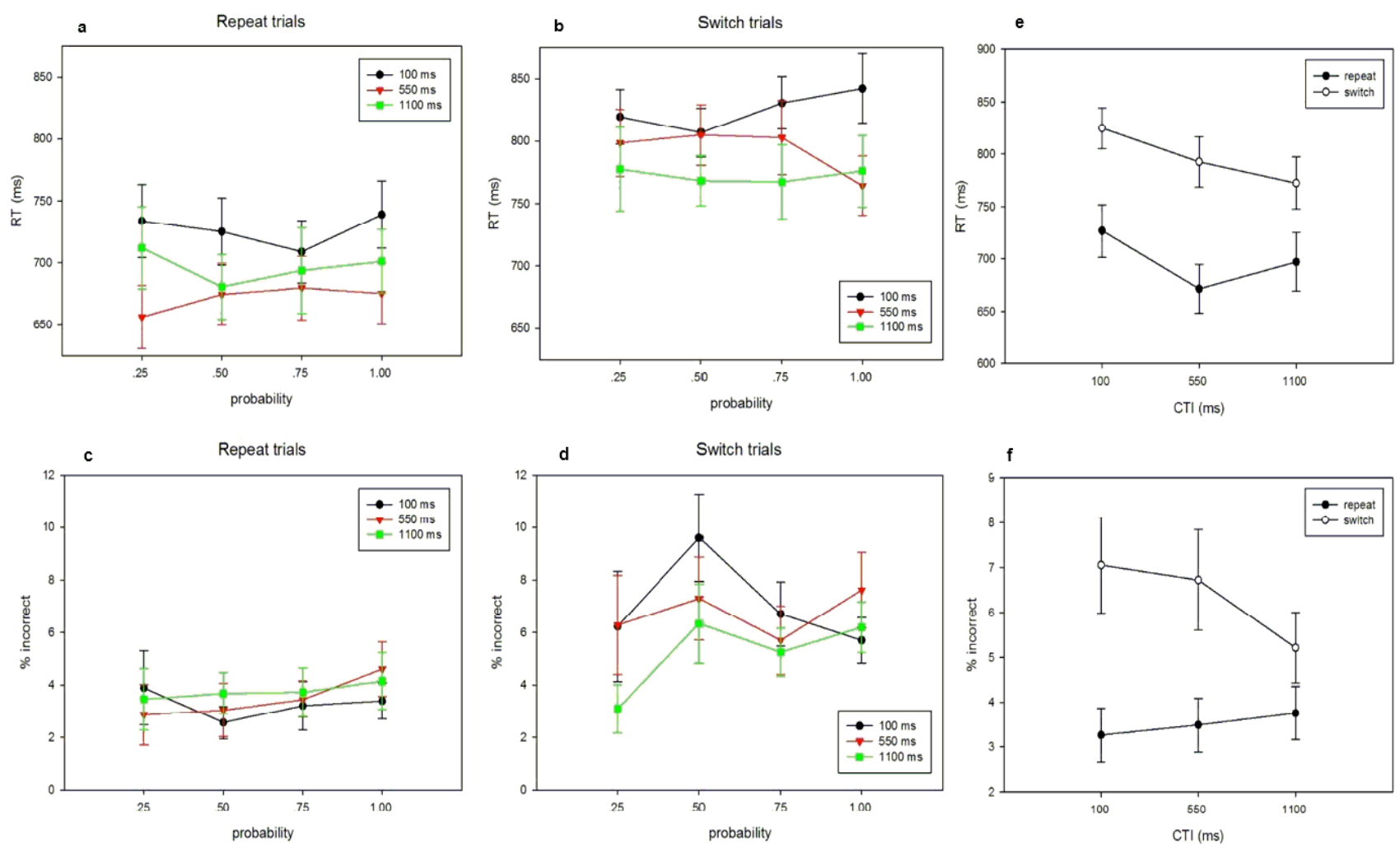

Figure 2. Quantifying behavioural responses for Experiment 1.

a, b. Mean RT as a function of probability, CTIs, and trial type (a for repeat, b for switch).

$c$, d. Mean error rate as a function of probability, CTIs, and trial type (c for repeat, $d$ for switch).

e. Mean RT as a function of CTI and trial type.

f. Mean error rate as a function of CTI and trial type.

Interestingly, neither significant main effects of probability on RT $(p=.701)$ or error rates $(p=.103)$ nor significant interaction effects of probability with CTI or trial type were found in this experiment. RTs and error rates were similar across all levels of probability in each combination of trial type and CTI (see Figures 2a-d). One possibility is that the abstract association between the cue and the task required a degree of experience with cues. To account for this, a re-analysis with ANOVA was conducted on the last five experimental blocks only, when participants had already been exposed to the same cue-task associations in 600 previous trials. Even in later blocks, probability did not have a significant effect on either RT, $p=.285$, or error rates, $p=.248$.

Another possibility is that participants varied in the amount of attention they paid to the contextual cues, as they were never explicitly mentioned as useful for the task and were technically redundant because of the explicit task cue. We used a measure of recognition memory for the background images as a proxy for how much attention participants paid to the contextual cues. As seen in Figure 3a, participants varied in the strength of their recognition memory. To control for differences in attention and memory for contextual cues, possible interactions between recognition memory score and the three independent variables were explored in separate 4 (probability) $\times 2$ (trial type $) \times 3(\mathrm{CTI})$ repeated measures ANCOVAs on RT and error rate, alpha $=.05$.

Recognition memory score did not correlate significantly with overall error rates $[r(16)=.18, p=.482]$, and while the pattern of results in Figure 3b-e suggests that participants with higher recognition memory scores were slightly faster $[r(16)=-.36, p=.141]$, this trend were not significant. Recognition score interacted with probability and CTI to significantly predict RT trends, $F(6,96)=2.41, p=.033, \eta^{2}=.13$. In low-probability trials (Figure 3b), low-recognition participants were slower after long CTIs $(1100 \mathrm{~ms})$ than after short CTIs (100 ms). Highrecognition participants, however, responded more quickly after long CTIs than after short CTIs. In high- 

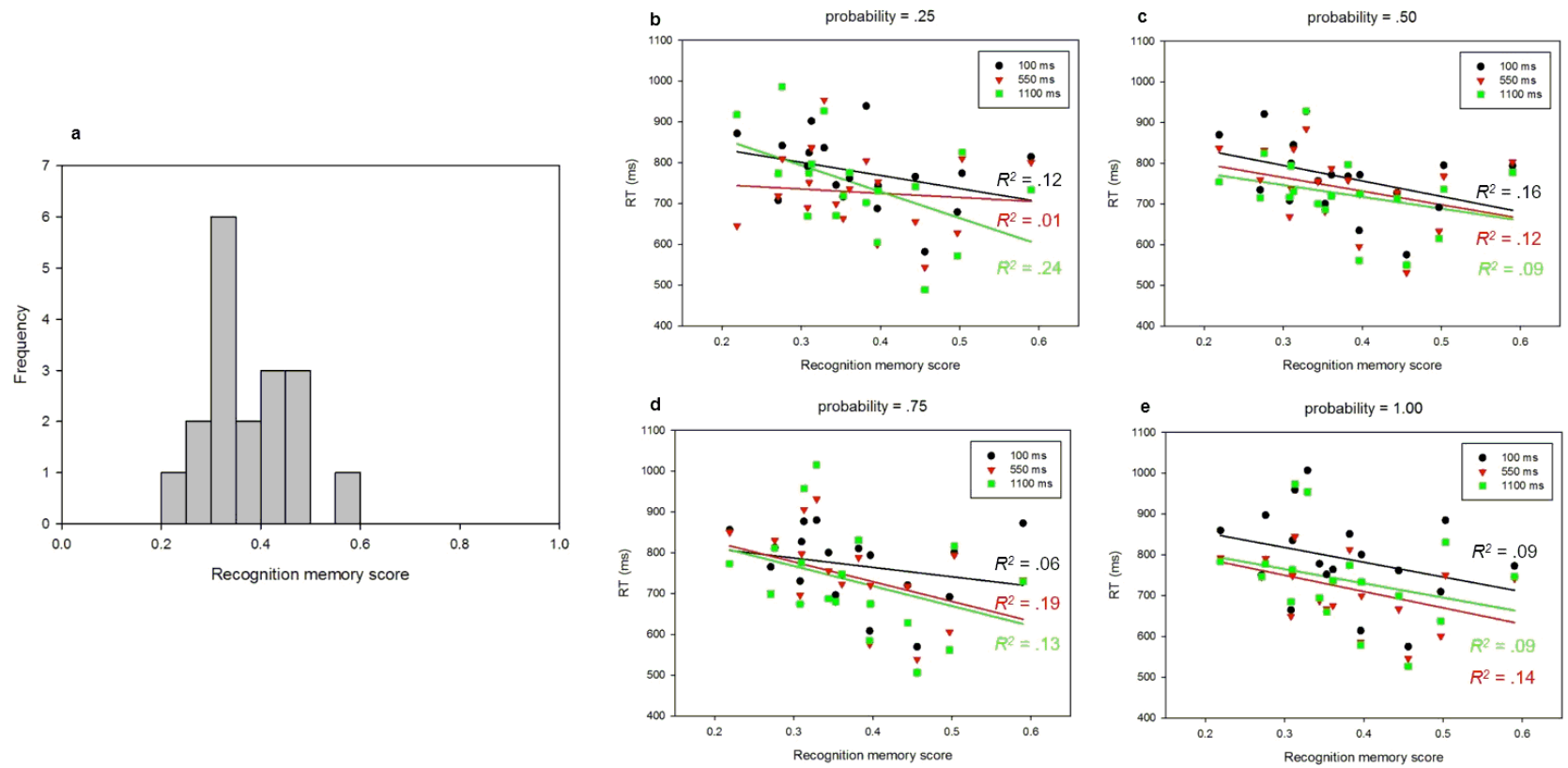

Figure 3. Individual differences in recognition memory for contextual cues and their effect on performance.

a. Distribution of recognition memory scores in Experiment 1. Recognition memory scores could technically fall between 0 and 1, but participants all scored between .22 and .59 . Scores were slightly right-skewed $(M=.37, M d n=$ $.36, S D=.09)$.

b, c, d, e. Mean RT as a function of recognition memory score, CTI, and probability.

probability trials (Figure 3d), low-recognition participants had very similar response times across all CTIs. Highrecognition participants, on the other hand, responded more quickly after medium (550 ms) and long (1100 ms) CTIs than after short $(100 \mathrm{~ms})$ CTIs. In both certain (Figure 3e) and indeterminate (Figure 3c) probability conditions, the relatively parallel nature of the three CTI lines reflected a main effect of CTI. No other significant interaction effects between the three independent variables and the covariate were found.

\section{Discussion}

In this experiment, participants were presented with implicit contextual cues that preceded and predicted the task stimuli; the category of the cue was associated with specific task probabilities. Switch costs and overall RT decreased as a function of CTI, but did not change as a function of cued task probability. However, the interaction effect of probability and CTI became significant once recognition memory scores for the image cues were taken into account: high-scoring participants tended to respond more quickly in general, and were faster after long CTI than after short CTI on low-probability trials. The results suggest that cues might have been used, but the strong probabilistic cueing effects that Dreisbach et al. (2002) observed were not found. There are two possible reasons. Most participants were unable to recall the five contextual categories at debrief, suggesting that the abstract nature of the cue-task association might have been challenging to learn implicitly. In addition, the presence of uninformative cues $(P=.50)$ might have deterred the learning of cue-task associations. These issues were addressed in Experiment 2.

\section{Experiment 2}

In Experiment 2, the contextually cued task-switching paradigm introduced in Experiment 1 was simplified. First, task probabilities were associated with specific contextual cues rather than contextual categories. In addition, only high- and low-probability conditions were used, as the interaction effects between probability, CTI, and 
recognition memory in Experiment 1 had been primarily driven by the high- $(P=0.75)$ and low- $(P=0.25)$ probability conditions. Finally, a separate cue-task association measure was added to quantify participants' explicit awareness of cue-task associations. Previous research has found that people may show an understanding of cue-task associations in their task performance without being explicitly aware of the cues or cue-task associations (e.g., Manly et al., 2014). These changes were made to (i) clarify the effects of probabilistic cues on task performance and (ii) determine whether these effects were being driven by explicit or implicit understanding of cue-task associations.

\section{Method}

Participants. Twenty-four fluently English-speaking volunteers recruited from the undergraduate and postgraduate student population at the University of Oxford took part in the experiment (16 female; $M=20.96$ years, $S D=1.71$ years, range 19-28 years). None of them had participated in Experiment 1. All participants had normal or corrected-to-normal vision, gave written informed consent prior to testing, and were reimbursed for their time with $£ 5$.

Apparatus and stimuli. All stimuli and apparatus remained the same as in Experiment 1, except the following specified changes. First, the frame colour for the left-right task was changed from blue (in Experiment 1) to yellow to discourage participants from assuming associations between the frame colour and the predominant colours in the background images (i.e., blue and green). The red frame remained unchanged as an explicit cue of the updown task. Second, during Experiment 2, target stimuli only appeared in the bottom-left or top-right quadrant because only target stimuli in these quadrants entailed different key responses for different tasks. Finally, only two background images were used throughout the practice and experimental blocks, and each was consistently associated with specific task probabilities ( $80 \%$ up-down / 20\% left-right for one image, and $20 \%$ up-down / 80\% left-right for the other).

Procedure. The experiment was conducted in a single session lasting 40 minutes. The format of individual trials was the same as in Experiment 1, but only CTIs of $100 \mathrm{~ms}$ and $1100 \mathrm{~ms}$ were used. After a practice block of 80 trials, participants completed 15 experimental blocks (40 trials each). No immediate post-trial feedback was given, but participants were presented with a summary of their mean RT and accuracy at the end of each block.

At the end of the experimental blocks, participants performed two surprise tests. The first was a cue recognition memory test. Ten images were shown: the two seen throughout the experiment and eight unseen. The unseen images were graded in terms of semantic (category) and perceptual (colour) similarity. Recognition memory score (possible range: 0 to 100) was calculated using the formula described in Supplementary Material (S2). The second was a cue-task association learning test. For the two seen images, participants were asked to indicate on separate sliding scales (i) which task (up-down or left-right) was more likely to follow the image ( $0 \%$ up-down $/ 100 \%$ left-right to $100 \%$ up-down $/ 0 \%$ left-right) and (ii) how confident they were ( $0 \%$ to $100 \%)$ in their cue-task association rating. Accuracy in cue-task probability estimates and retrospective confidence in those estimates were weighted in the calculation of an association learning score (possible range: 0 to 100) using the formula described in Supplementary Material (S3).

\section{Results}

The first trial of each block ( $2.5 \%$ of total) was excluded from both response time (RT) and error rate analyses. In addition, for RT analyses, trials on which errors were made ( $4.6 \%$ of total) and RT outliers ( $>3 S D$ from the mean or under $300 \mathrm{~ms}$ ) ( $1.8 \%$ of total) were identified for each participant and excluded. Mean RTs and error rates, and their respective switch costs, are summarized in Table 3 and 4, respectively.

RTs and error rates were analyzed in separate 2 (probability: $0.20,0.80) \times 2$ (trial type: repeat, switch) $\times 2$ (CTI: $100,1100)$ repeated measures ANOVA with alpha levels of .05 using SPSS v.22. Robust switch costs were observed for both RT and error rates. Participants responded more slowly on switch trials ( $895 \mathrm{~ms}$ ) than on repeat 
Table 3

Mean RT (ms) as a function of trial type, CTI, and probability in Experiment 2

\begin{tabular}{|c|c|c|c|c|c|c|}
\hline \multirow[b]{3}{*}{ CTI } & \multirow[b]{3}{*}{ Probability } & \multicolumn{4}{|c|}{ Trial type } & \multirow[b]{3}{*}{ Switch cost } \\
\hline & & \multicolumn{2}{|c|}{ Repeat } & \multicolumn{2}{|c|}{ Switch } & \\
\hline & & $M$ & $S E$ & $M$ & $S E$ & \\
\hline \multirow[t]{2}{*}{100} & .20 & 730 & 30 & 902 & 50 & 172 \\
\hline & .80 & 704 & 29 & 892 & 41 & 188 \\
\hline \multirow[t]{2}{*}{1100} & .20 & 781 & 43 & 893 & 55 & 112 \\
\hline & .80 & 751 & 37 & 892 & 51 & 141 \\
\hline
\end{tabular}

Table 4

Mean error rate (\% incorrect) as a function of trial type, CTI, and probability in Experiment 2

\begin{tabular}{|c|c|c|c|c|c|c|}
\hline \multirow[b]{3}{*}{ CTI } & \multirow[b]{3}{*}{ Probability } & \multicolumn{4}{|c|}{ Trial type } & \multirow[b]{3}{*}{ Switch cost } \\
\hline & & \multicolumn{2}{|c|}{ Repeat } & \multicolumn{2}{|c|}{ Switch } & \\
\hline & & $M$ & $S E$ & $M$ & $S E$ & \\
\hline \multirow[t]{2}{*}{100} & .20 & 2.2 & .7 & 7.1 & 1.3 & 4.9 \\
\hline & .80 & 2.0 & .4 & 5.5 & .8 & 3.5 \\
\hline \multirow[t]{2}{*}{1100} & .20 & 3.9 & .8 & 6.2 & 1.1 & 2.3 \\
\hline & .80 & 3.7 & .6 & 6.4 & .8 & 2.7 \\
\hline
\end{tabular}

trials $(742 \mathrm{~ms}), F(1,23)=49.48, p<.001, \eta^{2}=.68$, and they also made more errors on switch trials $(6.3 \%)$ than repeat trials $(3.0 \%), F(1,23)=22.14, p<.001, \eta^{2}=.49$. A significant main effect of probability was observed, $F(1,23)=6.72, p=.016, \eta^{2}=.23$. Responses did not differ significantly in accuracy between high-probability $(P$ $=.80)$ and low-probability trials $(P=.20), p=.258$, but were significantly slower on low-probability trials $(827$ $\mathrm{ms}$ ) than high-probability trials $(810 \mathrm{~ms})$. No significant interaction effects between probability and CTI or probability and trial type were found for RT or error rates. No significant main effect of CTI was observed for $\mathrm{RT}, p=.157$, or error rate, $p=.173$. However, the interaction effect of CTI and trial type was significant for RT, $F(1,23)=13.69, p=.001, \eta^{2}=.37$, and approached significance for error rates, $p=.055$. As seen in Figure 4a-b, switch costs decreased as a function of CTI because of poorer performance on repeat trials as CTI increased from $100 \mathrm{~ms}$ (717 ms, $2.1 \%$ error) to $1100 \mathrm{~ms}$ ( $766 \mathrm{~ms}, 3.8 \%$ error). Performance on switch trials did not differ greatly between short CTIs ( $897 \mathrm{~ms}, 6.3 \%$ error) and long CTIs ( $892 \mathrm{~ms}, 6.3 \%$ error).

As in Experiment 1, we checked for interactions between the independent variables and covariate measures of recognition and association learning. Although only two images were presented in the 600 trials of the experiment, a large variation in participants' recognition memory scores was still found (see Figure 4c). However, recognition memory score was not correlated with participants' performance in terms of $\mathrm{RT}, r(22)=.20, p=.339$, or error rates, $r(22)=-.11, p=.613$. No significant interactions between recognition memory scores and any of the independent variables were found in 2 (probability) $\times 2$ (trial type) $\times 2(\mathrm{CTI})$ repeated measures analyses of covariance on RT and error rate.

In this experiment, an association learning measure was introduced to quantify participants' explicit awareness of the cue-task associations. Participants' association learning scores also varied greatly (see Figure 4d) but were independent of their recognition memory scores, $r(22)=.05, p=.805$. As observed in Figure $4 \mathrm{~d}$, participants clustered into high-scoring $(M=66, S D=7, N=11)$ and low-scoring $(M=24, S D=11, N=13)$ subgroups. However, no significant differences in RT, $p=.660$, or error rates, $p=.993$, were found between the subgroups. The pattern of results suggested that RTs tended to be slightly slower for the high-score subgroup $(838 \mathrm{~ms})$ than 
a

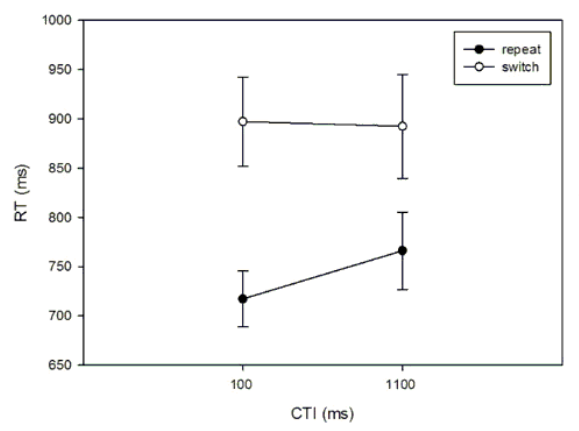

b

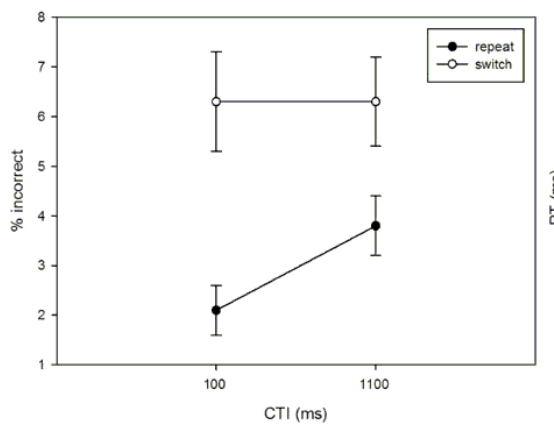

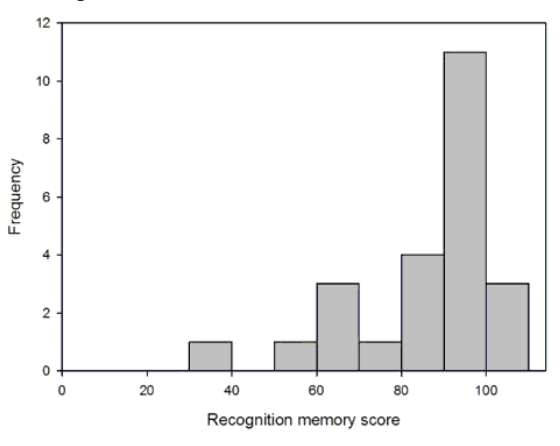

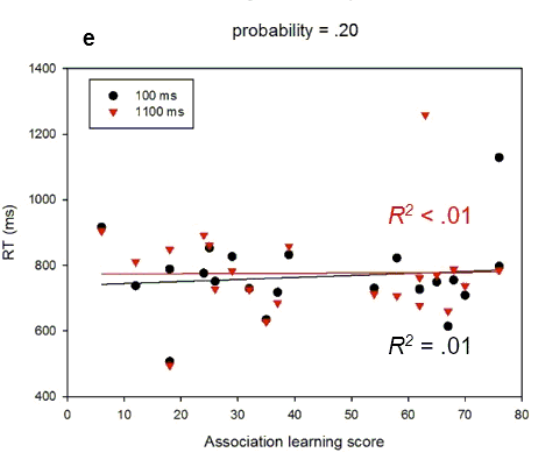

d

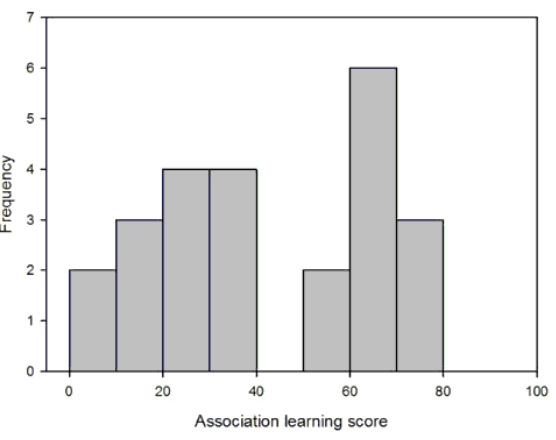

probability $=.80$

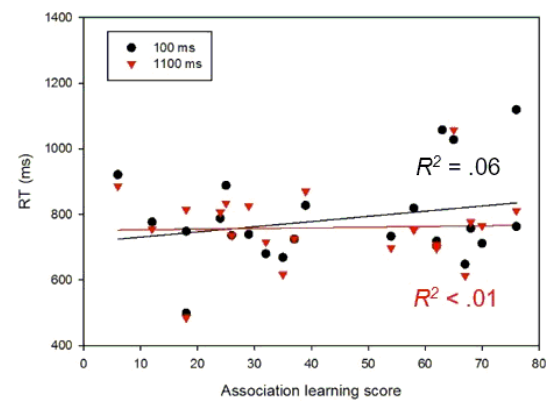

Figure 4. General trends and individual differences in behavioural responses.

a. Mean RT as a function of CTI and trial type.

b. Mean error rate as a function of CTI and trial type.

c. Distribution of recognition memory scores in Experiment 2. Recognition memory scores could technically fall between 0 and 100, but participants all scored between 30 and 100. Scores were left-skewed $(M=85, M d n=93, S D=$ 18).

d. Distribution of association learning scores in Experiment 2. Association learning scores could technically fall between 0 and 100, but participants all scored between 6 and 76. Scores were not normally distributed $(M=43, M d n$ $=38, S D=23$ ).

e-f. Mean RT as a function of association learning score, CTI, and probability. Outliers were identified as data points with a standardized residual greater than 2 , and were eliminated prior to fitting the regression lines.

the low-score subgroup (802 ms), while error rates for both subgroups were the same $(4.6 \%)$. When association learning scores were controlled for in separate 2 (probability) $\times 2$ (trial type) $\times 2$ (CTI) repeated measures analyses of covariance, no significant interactions between association learning scores and independent variables emerged, although the interaction effect of association learning, probability, and CTI approached significance, $p=.065$. Figure 4e-f suggests that, while there was no observable RT difference as a function of association learning scores at either CTI in low-probability trials, participants with high association learning scores may have tended to be slightly slower after short CTIs on high-probability trials. CTI did not seem to affect RTs on high-probability trials for participants with low association learning scores.

\section{Discussion}

In this experiment, we presented participants with implicit contextual cues that were directly associated with specific task probabilities. Participants were faster on high-probability than low-probability trials, showing probabilistic cueing effects similar to those found by Dreisbach et al. even though cue-task association were not explicitly instructed at any point. Unlike in Experiment 1, CTI did not affect overall RTs or error rates, although repeat trials were slower and less accurate after long CTIs than after short CTIs. Individual differences in recognition memory and association learning scores did not significantly interact with any of the independent variables, although the interaction effect of probability, CTI, and association learning score approached significance. This suggests that the cueing effect was mostly dissociable from explicit awareness of cue-task associations. 


\section{General Discussion}

The present study used implicitly cued task-switching designs, featuring probabilistic cues at different levels of abstractness, to address three research aims. Firstly, we wanted to test whether participants could implicitly learn probabilistic cue-task associations. We found some evidence for cue-task association learning, as reflected in a performance advantage for tasks that the contextual cues had indicated to be more probable. It is unclear whether this cueing effect is truly implicit or whether it could have been driven by some degree of explicit awareness of cue-task associations. Secondly, we wanted to compare the strength of the cueing effect when the cue-task associations were abstract versus concrete. Consistent with our hypothesis, the probabilistic cueing effect was only found when cue-task associations were concrete- that is, when each cue was associated with specific task probabilities. Finally, we wanted to assess the relative strength of top-down and bottom-up mechanisms engaged in this cueing paradigm. There was evidence for automatic co-activation of the competing task set: although we did not find greater switch costs for probabilistic cues relative to certain cues, participants with high recognition memory score for the contextual cues tended to respond faster after long CTI than after short CTI in lowprobability trials.

A performance advantage derived from implicit learning of cue-task associations was found, albeit not consistently across both experiments. On one hand, the results of Experiment 2 support the hypothesis that performance would be better for more probable tasks. Although error rates were similar, participants were considerably slower when confronted with a task less frequently associated with the preceding contextual cue. This effect is consistent with claims that stimuli acquire associations with tasks (Allport \& Wylie, 2000; Rogers \& Monsell, 1995; Waszak et al., 2003) and that people can spontaneously learn task structure (Collins \& Frank, 2013). Consistent with the results of Dreisbach et al. (2002), the strength of the RT advantage for high-probability trials did not vary between trial types (repeat and switch). On the other hand, no comparable probabilistic cueing effect was found in Experiment 1. Even in later blocks, when participants had considerable experience with the cues and tasks, they were still no faster or more accurate at responding to more probable tasks than less probable tasks.

This discrepancy in cue-associated performance advantage might be caused by a difference in experimental design and corresponding difficulty of learning. In Experiment 2, different background images were probabilistically associated with different tasks (concrete cue-task association), but in Experiment 1, different image categories were probabilistically associated with different tasks (abstract cue-task association). Learning the task probabilities associated with each contextual cue, as participants do in Experiment 2, would not have been a viable strategy in Experiment 1 due to the large number of different specific contextual cues used and the infrequent recurrence of each contextual cue in the experiment. Additional effort and processing time might be needed to extract meaningful task-relevant information (category) from the contextual cue and to generalise task set structure from specific learned associations. Thus, a bigger RT benefit from a longer cue processing interval might be expected when cue-task associations are abstract than when they are concrete. Consistent with this interpretation, RT in Experiment 1, but not Experiment 2, improved with longer CTIs and had a significant interaction effect with probability after accounting for individual differences in recognition memory score.

However, unlike the unconscious task priming effects found in Manly et al. (2014), explicit awareness of and control over the cueing effect cannot be ruled out in the present design. While better recognition for the images used as cues did not correlate with better performance in either experiment, participants in Experiment 2 with higher association learning scores, namely, those with greater explicit awareness of cue-task associations, tended to be slightly slower than their lower-scoring counterparts after short CTIs on high-probability trials. The performance of those with greater explicit cue-task association awareness was more disadvantaged by short task set preparation intervals, and is therefore more consistent with a top-down cognitive configuration account of task-switching commonly found in explicitly cued task-switching (Meiran, 1996; Rogers \& Monsell, 1995). But because the association learning score does not discriminate between explicit awareness of cue-task associations 
during the task and explicit awareness provoked upon reflection post-test, we cannot discern whether high-scorers were aware and treating the cues like explicit probabilistic cues during the task. The effects of explicit awareness on performance could be teased out by adding experimental blocks immediately after the cue-task association learning test, since the test also explicitly informs participants of the possibility of an association between the contextual cue and task. In these post-test blocks, we would expect a general shift towards faster responses on high-probability trials, especially after long CTIs, for both high- and low-scoring participants.

However, a stronger case might be made for attributing the implicit cueing effects observed in the present experimental design to bottom-up mechanisms. In the present two experiments, the probabilistic cueing effect on RT does not generally seem to vary with CTI. This is consistent with the suggestion that implicit cueing effects, because they engage bottom-up activation processes, are not affected by the length of the cue processing interval (Rubin \& Koch, 2006). In addition, interference from competing task sets seems to persist at long CTIs in the present study. On low-probability trials in Experiment 1, participants with higher recognition scores responded faster after long CTI than after short CTI. This finding contradicts the claim that long-CTI trials are immune from any bottom-up influence, and suggests stronger bottom-up effects than found in the original implicitly cued taskswitching design (Rubin and Koch, 2006).

At first glance, this finding might also be rather counterintuitive, as the expectation is that participants prepare for the more likely task during the long CT and would therefore have much slower RT when unexpectedly confronted with a low-probability task that they did not prepare for. But the assumption underlying this expectation-that only one task set can be activated at any one time-might be questioned. It has been suggested that stimuli may automatically activate all associated task sets in previous experience, even unlikely ones (Allport \& Wylie, 2000; Rogers \& Monsell; Waszak et al., 2003). The contextual cues in the present study may therefore have concurrently activated both associated task sets. Based on existing multiple-component models of task-switching (e.g., Mayr \& Kliegl, 2003; Schmitz \& Voss, 2014) and evidence of how multiple response options are represented neurally (e.g., Cisek \& Kalaska, 2005), we hypothesize that concurrent activation of two task sets could unfold in the manner of Figure 5.

As illustrated in the schematic, the probabilistic task cue that occurs at time point $t_{0}$ activates both task sets it has been previously associated with (A and B). Based on experience, Task A has the higher probability (represented by the slope of the line) of following that cue than Task B, so Task Set A reaches activation threshold faster. When a task set reaches activation threshold, it initiates what we would call an action plan: a top-down process of configuring cognitive resources in preparation for the execution of a particular response (Monsell, 2003). The more cognitive resources dedicated to an action plan, the more detailed the action plan, and the faster and smoother the response is carried out once initiated by the explicit cue. However, if both task sets reach the activation threshold, the shared pool of cognitive resources means that a more detailed representation of the action plan for one task and its corresponding response might come at the expense of the other. The degree to which cognitive resources are allocated or prioritised may be strongly influenced by the ratio of the number of prior trials implementing one or the other in response to the same stimuli (Wylie \& Allport, 2000). If, as in Experiment 2, there is an $80 \%$ chance of Task A and a $20 \%$ chance of Task B, we would expect the action plan for Task A to be allocated more of the total available pool of cognitive resources than Task B. Based on this model, if a response is called for by the explicit cue at time point $t_{l}$ (when Task Set A is activated but Task Set B is not), we would expect a fast response for Task A, because of the activated action plan, but a very slow response for Task B, because the task set has not yet reached activation to initiate the corresponding action plan. If a response is called for at time point $t_{2}$ (when both Task Set A and Task Set B are activated), we would still expect a faster response for Task A because its action plan is represented in more detail. However, an interference effect for Task A would be seen when comparing RT at $t_{1}$ and $t_{2}$ due to competing cognitive processes for the alternate action plan being present at $t_{2}$ but not $t_{1}$. By comparison, responses for Task B would be consistently faster at $t_{2}$ than at $t_{l}$ due to the initiation of its action plan. This is exactly what we observed among participants with high recognition scores in Experiment 1: performance for the unexpected task (Task B) was better after long CTIs $\left(t_{2}\right)$ than short CTIs $\left(t_{1}\right)$. 


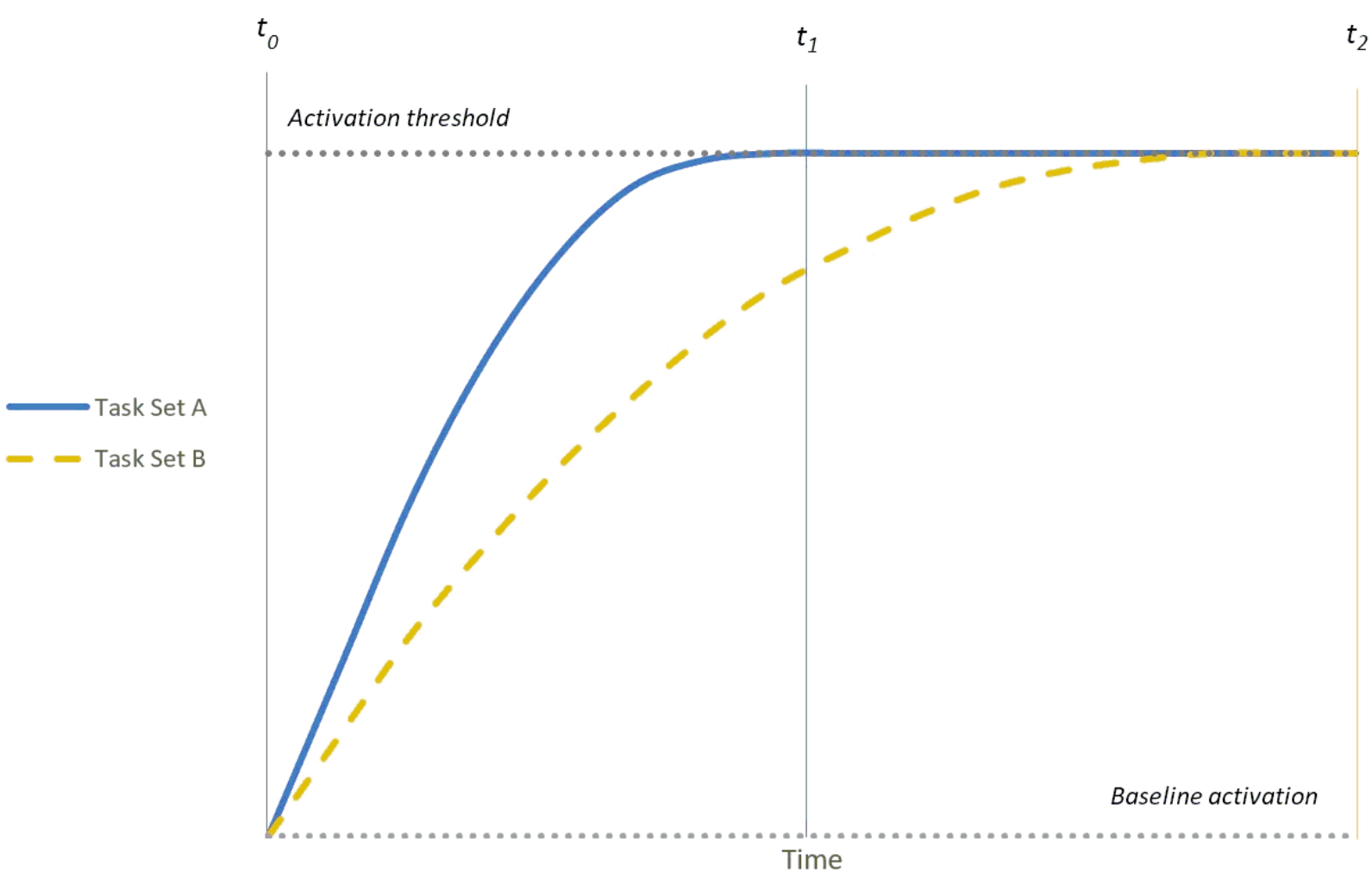

Figure 5. Schematic of multiple concurrent task set activation processes on each trial of the probabilistically cued taskswitching paradigm.

This model and the findings in the present study may have practical implications for improving situational awareness and multitasking performance in many real-world settings. Our findings build upon earlier situational awareness research by suggesting that people can adapt their performance in response to information from contextual cues. The model suggests implicit and probabilistic cues present in the context trigger cognitive representations of all possible scenarios and the dominant response in each of these scenarios. However, this capacity to represent multiple tasks or response options simultaneously, which could also underlie human multitasking ability, may be bound by limited cognitive resources. More time to process contextual cues can help us prepare for and improve our response to both high- and low-probability events, while increasing exposure to low-probability events and their associated context may improve response times to unexpected events in particular. This explains why, with more experience and resultant implicit learning of context-task associations, drivers are better able to predict and respond to unexpected situations, or situations that require a response other than the dominant one. It makes a case for the importance of simulation training for those who require rapid and high-complexity performance despite unexpected situations, like operating room staff and emergency responders. While additional evidence is needed to evaluate the proposed mechanisms of contextual influence and the transfer of these findings to real-world situations, the present study brings us closer to understanding how we might extract and use information from contexts to guide our behaviour in dynamic, uncertain environments.

\section{Ethical compliance}

All procedures involving human participants in the two experiments were approved by the Central University Research Ethics Committee of the Medical Sciences Division at the University of Oxford (MS-IDREC-C1-2015221) and comply with the British Psychological Society Ethical Standards and with the 1964 Helsinki Declaration and its later amendments. 


\section{Acknowledgments}

This research was supported by an undergraduate research grant awarded to LELW by Magdalen College, Oxford. The views and conclusions contained in this document are those of the authors and do not necessarily reflect the views of the funding agencies. The authors declare no competing financial interests.

LELW and NY designed the experiments. LELW ran the experiments and analysed data. LELW and NY wrote the manuscript. The authors also wish to thank Lucy Bowes and Shining Sun for helpful discussions in the design of this research, and Oscar Darwin, Eleanor Gale, and Samuel Kim for assistance with technical aspects of the experimental design.

\section{References}

Allport, A., Styles, E. A., \& Hsieh, S. (1994). Shifting intentional set: Exploring the dynamic control of tasks. In C. Umiltà \& M. Moscovitch (Eds.), Attention and Performance XV (pp. 421-452). Cambridge, MA: MIT Press.

Allport, A., \& Wylie, G. (2000). Task-switching, stimulus-response bindings, and negative priming. In S. Monsell \& J. S. Driver (Eds.), Control of cognitive processes: Attention and Performance XVIII (pp. 35-70). Cambridge, MA: MIT Press.

Altmann, E. M., \& Gray, W. D. (2008). An integrated model of cognitive control in task switching. Psychological Review, 115, 602-639.

Arbuthnott, K. D., \& Woodward, T. S. (2002). The influence of cue-task association and location on switch cost and alternating-switch cost. Canadian Journal of Experimental Psychology, 56, 18-29.

Arrington, C. M., \& Logan, G. D. (2005). Voluntary task switching: Chasing the elusive homunculus. Journal of Experimental Psychology: Learning, Memory, and Cognition, 31(4), 683-702.

Chun, M. M., \& Jiang, Y. (1998). Contextual cueing: Implicit learning and memory of visual context guides spatial attention. Cognitive Psychology, 36, 28-71.

Cisek, P., \& Kalaska, J. F. (2005). Neural correlates of reaching decisions in dorsal prefrontal cortex: Specification of multiple direction choices and final selection of action. Neuron, 45, 801-814.

Cleeremans, A., Destrebecqz, A., \& Boyer, M. (1998). Implicit learning: news from the front. Trends in Cognitive Sciences, 2(10), 406-416.

Collins, A. G. E., \& Frank, M. J. (2013). Cognitive control over learning: creating, clustering, and generalizing task-set structure. Psychological Review, 120, 190-229.

Craik, F. I. M. (1983). On the transfer of information from temporary to permanent memory. Philosophical Transactions of the Royal Society of London, Series B, 302, 341-359.

Crump, M. J. C., \& Logan, G. D. (2010). Contextual control over task-set retrieval. Attention, Perception \& Psychophysics, 72, 2047-2053.

Crump, M. J. C., Vaquero, J. M. M., \& Milliken, B. (2008). Context-specific learning and control: The roles of awareness, task relevance, and relative salience. Consciousness and Cognition, 17, 22-36.

Dreisbach, G., Haider, H., \& Kluwe, R. H. (2002). Preparatory processes in the task-switching paradigm: Evidence from the use of probability cues. Journal of Experimental Psychology: Learning, Memory, and Cognition, 28(3), 468-483.

Forrest, C. L. D., Monsell, S., \& McLaren, I. P. L. (2014). Is performance in task-cuing experiments mediated by task set selection or associative compound retrieval? Journal of Experimental Psychology: Learning, Memory, and Cognition, 40, 1002-1024.

Goujon, A. (2011). Categorical implicit learning in real-world scenes: Evidence from contextual cueing. The Quarterly Journal of Experimental Psychology, 64, 920-941.

Kunar, M. A., Flusberg, S. J., \& Wolfe, J. M. (2006). Contextual cueing by global features. Perception \& Psychophysics, 68(7), 1204-1216. 
Leue, A., Rodilla, C. C., \& Beauducel, A. (2015). The context matters: outcome probability and expectation mismatch modulate the feedback negativity when self-evaluation of response correctness is possible. BioMed Research International, 2015, 726798.

Lindenberger, U., \& Mayr, U. (2014). Cognitive aging: Is there a dark side to environmental support? Trends in Cognitive Sciences, 18, 7-15.

Logan, G. D. (2003). Executive control of thought and action: In search of the wild homunculus. Current Directions in Psychological Research, 12, 45-48.

Logan, G. D., \& Bundesen, C. (2003). Clever homunculus: Is there an endogenous act of control in the explicit task cuing procedure? Journal of Experimental Psychology: Human Perception and Performance, 29, 579-599.

Logan, G. D., \& Bundesen, C. (2004). Very clever homunculus: Compound stimulus strategies for the explicit task-cuing procedure. Psychonomic Bulletin, 11(5), 832-840.

Manly, T., Fish, J. E., Griffiths, S., Molenveld, M., Zhou, F. A., \& Davis, G. J. (2014). Unconscious priming of task-switching generalizes to an untrained task. PLoS ONE, 9(2), e88416. doi:10.1371/journal.pone.0088416

Mayr, U. (2002). Inhibition of action rules. Psychonomic Bulletin \& Review, 9, 93-99.

Mayr, U. (2006). What matters in the cued task-switching paradigm: tasks or cues? Psychonomic Bulletin and Review, 13(5), 794-799.

Mayr, U., \& Bryck, R. L. (2005). Outsourcing control to the environment: effects of stimulus/response locations on task selection. Psychological Research, 71, 107-116.

Mayr, U., \& Bryck, R. L. (2006). Sticky rules: Integration between abstract rules and specific actions. Journal of Experimental Psychology: Learning, Memory and Cognition, 31, 337-350.

Mayr, U., \& Keele, S. W. (2000). Changing internal constraints on action: The role of backward inhibition. Journal of Experimental Psychology: General, 129, 4-26.

Mayr, U., \& Kliegl, R. (2003). Differential effects of cue changes and task changes on task-set selection costs. Journal of Experimental Psychology: Learning, Memory, and Cognition, 29(3), 362-372.

McGuinness, B., \& Dawson, B. (2005). Assessing situational awareness in teams. In P. D. Bust \& P. T. McCabe (Eds.), Contemporary Ergonomics 2005: Proceedings of the International Conference on Contemporary Ergonomics (CE2005), 5-7 April 2005, Hatfield, UK (pp. 84-88). UK: Taylor \& Francis.

Meier, C., Lea, S. E. G., \& McLaren, I. P. L. (2016). Task-switching in pigeons: Associative learning or executive control? Journal of Experimental Psychology: Animal Learning and Cognition, 42, 163-176.

Meiran, N. (1996). Reconfiguration of processing mode prior to task performance. Journal of Experimental Psychology: Learning, Memory, and Cognition, 22(6), 1423-1442.

Monsell, S. (2003). Task switching. Trends in Cognitive Sciences, 7(3), 134-140.

Norman, D. A., \& Shallice, T. (1980). Attention to action: Willed and automatic control of behaviour (Technical Report No. 99). Center for Human Information Processing.

Norman, D. A., \& Shallice, T. (1986). Attention to action: Willed and automatic control of behaviour. In R. J. Davidson, G. E. Schwartz, \& D. Shapiro (Eds.), Consciousness and self-regulation (pp. 1-18). New York, NY: Plenum Press.

O’Brien, K. S., \& O'Hare, D. (2007). Situational awareness ability and cognitive skills training in a complex real-world task. Ergonomics, 50(7), 1064-1091.

Patterson, R. E., Pierce, B. J., Boydstun, A. S., Ramsey, L. M., Shannan, J., Tripp, L., \& Bell, H. (2013). Training intuitive decision making in a simulated real-world environment. Human Factors, 55, 333345.

Pierce, J. W. (2007). PsychoPy—Psychophysics software in Python. Journal of Neuroscience Methods, $162,8-13$.

Rogers, R. D., \& Monsell, S. (1995). Costs of a predictable switch between simple cognitive tasks. Journal of Experimental Psychology: General, 124(2), 207-231.

Rubin, O., \& Koch, I. (2006). Exogenous influences on task set activation in task switching. The Quarterly Journal of Experimental Psychology, 59(6), 1033-1046. 
Ruthruff, E., Remington, R. W., \& Johnston, J. C. (2001). Switching between simple cognitive tasks: the interaction of top-down and bottom-up factors. Journal of Experimental Psychology: Human Perception and Performance, 27, 1404-1419.

Schmitz, F., \& Voss, A. (2014). Components of task switching: A closer look at task switching and cue switching. Acta Psychologia, 151, 184-196.

Seger, C. A. (1994). Implicit learning. Psychological Bulletin, 115(2), 163-196.

Sudevan, P., \& Taylor, D. A. (1987). The cuing and priming of cognitive operations. Journal of Experimental Psychology: Human Perception and Performance, 13, 89-103.

Waszak, F., Hommel, B., \& Allport, A. (2003). Task-switching and long-term priming: Role of episodic stimulus-task bindings in task-shift costs. Cognitive Psychology, 46, 361-413.

Wylie, G., \& Allport, A. (2000). Task switching and the measurement of "switch costs". Psychological Research, 63, 212-233. 


\section{SUPPLEMENTARY MATERIAL}

\section{S1: Recognition memory score (Experiment 1)}

Frequencies of each rating were tallied up separately for images seen and not seen during the experiment according to the categories in Table A. The maximum recognition memory score is 1 (if all seen images were rated as +3 and all unseen images as -3 ). The minimum score is 0 (if all seen and unseen images are rated as -3 ).

Table A

Recognition memory score tally for a sample participant in Experiment 1

\begin{tabular}{cccc}
\hline Rating & $\begin{array}{c}\text { Exact image seen during } \\
\text { experiment }\end{array}$ & $\begin{array}{c}\text { Category X not seen } \\
\text { (same category as a seen } \\
\text { image })\end{array}$ & $\begin{array}{c}\text { Category Y not seen } \\
\text { (different category from } \\
\text { all seen image) }\end{array}$ \\
\hline-3 & 4 & 3 & 11 \\
-2 & 20 & 6 & 3 \\
-1 & 10 & 1 & 0 \\
+1 & 10 & 4 & 1 \\
+2 & 7 & 1 & 0 \\
\hline 3 & 9 & 0 & 0 \\
\hline Total frequency & 60 & 15 & 15 \\
\hline
\end{tabular}

The raw recognition memory score was calculated as the sum of the weighted frequencies (see Table B for assigned weights). The raw score was weighted so that hit rates for seen images were more influential and false alarm rates for unseen images varied depending on their similarity to seen images.

Table B

Weight matrix for recognition memory scores in Experiment 1

\begin{tabular}{cccc}
\hline Rating & $\begin{array}{c}\text { Exact image seen during } \\
\text { experiment }\end{array}$ & Category X not seen & Category Y not seen \\
\hline-3 & -3 & 3.5 & 3 \\
-2 & -2 & 2.5 & 2 \\
-1 & -1 & 1.5 & 1 \\
+1 & 1 & -1 & -1.5 \\
+2 & 2 & -2 & -2.5 \\
\hline
\end{tabular}

For instance, the raw score for our sample participant would be calculated as follows:

\begin{tabular}{l|l|l} 
Category & Frequency $\times$ weight & Raw score subtotal \\
\hline Seen images & $4 \times(-3)+20 \times(-2)+10 \times(-1)+10 \times(1)+7 \times(2)+9 \times(3)$ & -11 \\
\hline $\begin{array}{l}\text { Unseen images } \\
(\text { Category X) }\end{array}$ & $3 \times(3.5)+6 \times(2.5)+1 \times(1.5)+4 \times(-1)+1 \times(-2)+0 \times(-3)$ & 21 \\
\hline $\begin{array}{l}\text { Unseen images } \\
(\text { Category Y) }\end{array}$ & $11 \times(3)+3 \times(2)+0 \times(1)+1 \times(-1.5)+0 \times(-2.5)+0 \times(-3.5)$ & 37.5 \\
\hline \multicolumn{2}{r}{ Raw recognition memory score: } & 47.5
\end{tabular}

The raw score (range: -277.5 to +277.5 ) was scaled down using the formula:

$$
\text { Recognition memory score }=\frac{82.5+\text { raw score }}{360}
$$

(Negative values returned by the formula are converted into a score of 0 .) 
Our sample participant, according to this conversion formula, would have a recognition score of .361 on a scale of 0 to 1.

\section{S2: Recognition memory score (Experiment 2)}

Frequencies of each rating were tallied up separately for images seen and not seen during the experiment according to the categories in Table $\mathrm{C}$. The maximum recognition memory score is 100 (if all seen images were rated as +3 and all unseen images as -3 ). The minimum score is 0 (if all seen images were rated as -3 and all unseen images as +3$)$.

Table C

Recognition memory score tally for a sample participant in Experiment 2

\begin{tabular}{cccccc}
\hline Rating & $\begin{array}{c}\text { Exact image } \\
\text { seen during } \\
\text { experiment }\end{array}$ & $\begin{array}{c}\text { Category A not } \\
\text { seen }\end{array}$ & $\begin{array}{c}\text { Category B not } \\
\text { seen }\end{array}$ & $\begin{array}{c}\text { Category C not } \\
\text { seen }\end{array}$ & $\begin{array}{c}\text { Category D not } \\
\text { seen }\end{array}$ \\
\hline-3 & 0 & 1 & 2 & 2 & 2 \\
-2 & 0 & 1 & 0 & 0 & 0 \\
-1 & 0 & 0 & 0 & 0 & 0 \\
+1 & 0 & 0 & 0 & 0 & 0 \\
+2 & 0 & 0 & 0 & 0 & 0 \\
+3 & 2 & 0 & 0 & 0 & 2 \\
\hline Total frequency & 2 & 2 & 2 & 2 & \\
\hline
\end{tabular}

The raw recognition memory score was calculated as the sum of the weighted frequency (see Table D for assigned weights). The raw score was weighted so that hit rates for seen images were more influential, and false alarm rates for unseen images varied depending on their similarity to seen images.

Table D

Weight matrix for recognition memory scores in Experiment 2

\begin{tabular}{cccccc}
\hline Rating & Seen & $\begin{array}{c}\text { Category A } \\
\text { unseen }\end{array}$ & $\begin{array}{c}\text { Category B } \\
\text { unseen }\end{array}$ & $\begin{array}{c}\text { Category C } \\
\text { unseen }\end{array}$ & $\begin{array}{c}\text { Category D } \\
\text { unseen }\end{array}$ \\
\hline-3 & -300 & 35 & 34 & 31 & 30 \\
-2 & -200 & 25 & 24 & 21 & 20 \\
-1 & -100 & 15 & 14 & 11 & 10 \\
+1 & 100 & -10 & -11 & -14 & -15 \\
+2 & 200 & -20 & -21 & -24 & -25 \\
+3 & 300 & -30 & -31 & -34 & -35 \\
\hline
\end{tabular}

For instance, the raw score for our sample participant would be calculated as follows:

\begin{tabular}{l|l|l} 
Category & Calculation & $\begin{array}{l}\text { Raw score } \\
\text { subtotal }\end{array}$ \\
\hline Seen images & $0 \times(-300)+0 \times(-200)+0 \times(-100)+0 \times(100)+0 \times(200)+2 \times(300)$ & 600 \\
\hline $\begin{array}{l}\text { Unseen images } \\
(\text { Category A })\end{array}$ & $1 \times(35)+1 \times(25)+0 \times(15)+0 \times(-10)+0 \times(-20)+0 \times(-30)$ & 60 \\
\hline $\begin{array}{l}\text { Unseen images } \\
\text { (Category B })\end{array}$ & $2 \times(34)+0 \times(24)+0 \times(14)+0 \times(-11)+0 \times(-21)+0 \times(-31)$ & 68 \\
\hline $\begin{array}{l}\text { Unseen images } \\
(\text { Category C) }\end{array}$ & $2 \times(31)+0 \times(21)+0 \times(11)+0 \times(-14)+0 \times(-24)+0 \times(-34)$ & 62 \\
\hline $\begin{array}{l}\text { Unseen images } \\
(\text { Category D) }\end{array}$ & $2 \times(30)+0 \times(20)+0 \times(10)+0 \times(-15)+0 \times(-25)+0 \times(-35)$ & 60 \\
\hline \multicolumn{2}{r}{ Raw recognition memory score: } & 850
\end{tabular}


The raw score (range: -860 to +860 ) was scaled down using the formula:

$$
\text { Recognition memory score }=\frac{860+\text { raw score }}{17.2}
$$

Our sample participant, according to this conversion formula, would have a recognition score of 99.42 on a scale of 0 to 100 .

\section{S3: Association learning score (Experiment 2)}

The cue-task association scale was coded in terms of the probability of the left-right task [ $P$ (yellow)] following the image: 100 indicated that the left-right task always followed the image, 0 indicated that the up-down task always followed the image, and 50 indicated that either task was equally likely to follow. On the confidence scale, 100 indicated certainty in the probability estimate, and 0 indicated complete uncertainty.

The maximum association learning score is 100 [if rated $P($ yellow $)=$ actual $P($ yellow $)$ and rated confidence was 100 for both seen images]. The minimum score is 0 (if rated confidence $=0$ for both images, regardless of the ratings for task probabilities).

Raw association learning scores for the two seen images was calculated as:

$$
0.1 \times \sum_{\text {image }}\left(\text { rated confidence } \times \frac{100-\mid \text { rated } P(\text { yellow })-\text { actual } P(\text { yellow }) \mid}{10}\right)
$$

The raw score (range: 0 to 200) was scaled down by a factor of 0.5 to produce the association learning score.

For instance, a participant who rated $P($ yellow $)=75$ with a confidence of 70 for one image with an actual $P($ yellow $)=80$, and rated $P($ yellow $)=30$ with a confidence of 70 for the other image with an actual $P($ yellow $)=$ 20 , the raw association learning score would be:

$$
0.1 \times\left(70 \times \frac{100-|75-80|}{10}+70 \times \frac{100-|30-20|}{10}\right)=129.5
$$

Our sample participant, according to the conversion formula, would have an association learning score of 64.75 on a scale of 0 to 100 . 